\title{
Frequency division transmission imaging and synthetic aperture reconstruction
}

\section{Gran, Fredrik; Jensen, Jørgen Arendt}

\section{Published in:}

I E E E Transactions on Ultrasonics, Ferroelectrics and Frequency Control

Link to article, DOI:

10.1109/TUFFC.2006.1632681

Publication date:

2006

Document Version

Publisher's PDF, also known as Version of record

Link back to DTU Orbit

Citation (APA):

Gran, F., \& Jensen, J. A. (2006). Frequency division transmission imaging and synthetic aperture reconstruction. I E E E Transactions on Ultrasonics, Ferroelectrics and Frequency Control, 53(5), 900 - 911. https://doi.org/10.1109/TUFFC.2006.1632681

\section{General rights}

Copyright and moral rights for the publications made accessible in the public portal are retained by the authors and/or other copyright owners and it is a condition of accessing publications that users recognise and abide by the legal requirements associated with these rights.

- Users may download and print one copy of any publication from the public portal for the purpose of private study or research.

- You may not further distribute the material or use it for any profit-making activity or commercial gain

- You may freely distribute the URL identifying the publication in the public portal 


\title{
Frequency Division Transmission Imaging and Synthetic Aperture Reconstruction
}

\author{
Fredrik Gran and Jørgen Arendt Jensen, . .
}

\begin{abstract}
In synthetic transmit aperture imaging only a few transducer elements are used in every transmission, which limits the signal-to-noise ratio (SNR). The penetration depth can be increased by using all transmitters in every transmission. In this paper, a method for exciting all transmitters in every transmission and separating them at the receiver is proposed. The coding is done by designing narrow-band linearly frequency modulated signals, which are approximately disjointed in the frequency domain and assigning one waveform to each transmitter. By designing a filterbank consisting of the matched filters corresponding to the excitation waveforms, the different transmitters can be decoded at the receiver. The matched filter of a specific waveform will allow information only from this waveform to pass through, thereby separating it from the other waveforms. This means that all transmitters can be used in every transmission, and the information from the different transmitters can be separated instantaneously. Compared to traditional synthetic transmit aperture (STA) imaging, in which the different transmitters are excited sequentially, more energy is transmitted in every transmission, and a better signal-to-noise-ratio is attained. The method has been tested in simulation, in which the resolution and contrast was compared to a standard synthetic transmit aperture system with a single sinusoid excitation. The resolution and contrast was comparable for the two systems. The method also has been tested using the experimental ultrasound scanner RASMUS. The resolution was evaluated using a string phantom. The method was compared to a conventional STA using both sinusoidal excitation and linear frequency modulated (FM) signals as excitation. The system using the FM signals and the frequency division approach yielded the same performance concerning both axial $($ of $\approx 3 \lambda)$ and lateral resolution (of $\approx 4.5 \lambda$ ). A SNR measurement showed an increase in SNR of $6.5 \mathrm{~dB}$ compared to the system using the conventional STA method and FM signal excitation.
\end{abstract}

\section{INTRODUCTION}

$\mathrm{N}$ ORMAL linear array images are made from a set of beamformed lines. Each line is created by transmitting a focused beam along a specific direction, and beamforming the received echoes from the receiving transducer elements using either fixed or dynamic focus. By applying this method, one image line will be created per transmission, making the method sensitive to motion.

Manuscript received August 9, 2004; accepted October 11, 2005. This work was supported by grant 9700883,9700563 and $26-01-0178$ from the Danish Science Foundation and by B-K Medical A/S, Denmark.

The authors are with the Center for Fast Ultrasound Imaging, Ørsted•DTU, Technical University of Denmark, 2800 Kgs. Lyngby, Denmark (e-mail: fg@oersted.dtu.dk).
In synthetic transmit aperture imaging [1], [2], only one element is used in every transmission. By this approach, a spherical wave is created that excites the tissue spreading the acoustic energy in all directions in contrast to focusing the energy in a single point. Instead of beamforming only one line, an entire image can be formed because information from all directions is available. Perfect focus, thus, can be attained for every point in the image because the origin for the emanating wave is known, as well as where the propagating wave was received. Because only a single element is used as the transmitting aperture, the resolution in the resulting image will be poor [3]. However, by transmitting from several different locations on the aperture and summing the individual images, the full aperture can be synthesized. The major advantage for the STA approach is clearly the ability to produce perfect focusing for all points in the image. A major disadvantage, however, is the poorer signal-to-noise ratio (SNR), which results from that only a single element is used at a time and the acoustic energy is distributed all over the tissue. Furthermore, the process of synthesizing the aperture rests on the assumption that the propagating medium is stationary, which can impose a problem in certain applications in medical ultrasound, e.g., flow imaging and imaging rapidly moving tissue.

The problem concerning SNR was addressed previously in [4]-[8] in which longer linear frequency modulated (FM) signals were transmitted, increasing the transmitted energy without increasing the instantaneous amplitude of the signals. Another way of increasing the SNR is by grouping a number of transducer elements and focusing close to the transducer surface. This can be achieved either by focusing in front or behind the transducer, creating a defocused high energetic virtual source. This was proposed in [9]-[12] and studied further in [13], [14]. Recently a clinical study was made combining the use of virtual sources and linear FM signals and comparing the results to conventional convex array imaging [15]. By applying virtual sources, a larger portion of the aperture is used, but the full aperture is still not used in every transmission. In [16], [17], a Hadamard encoding scheme was proposed, so that the full aperture can be excited at the same time. This approach, however, requires $N$ transmissions in order to decode the information provided that $N$ transmitters ${ }^{1}$ are used. Another approach to spatio-temporal coding was proposed in [18] using orthogonal Golay coded excitation. This method also requires more than one transmission before the infor-

\footnotetext{
${ }^{1}$ The term transmitter will be used to denote a transducer element or a virtual source.
} 
Transmitting aperture
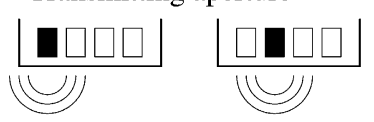

Receiving aperture

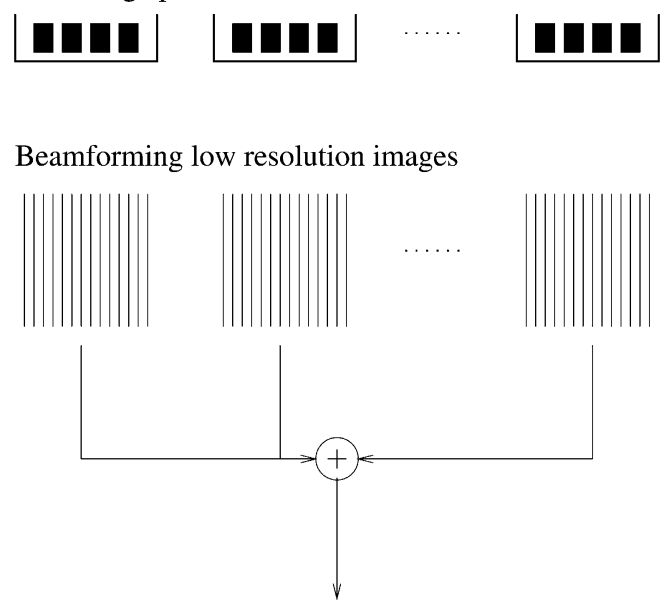

High resolution image
Transmitting aperture
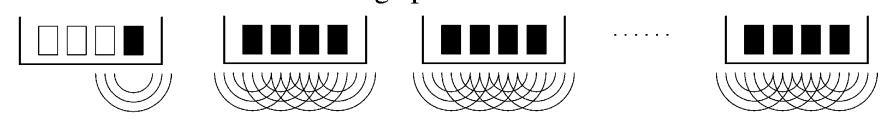

Receiving aperture

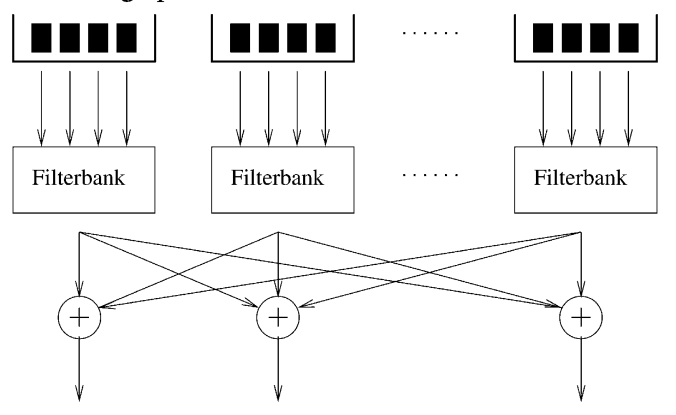

Beamforming low resolution images

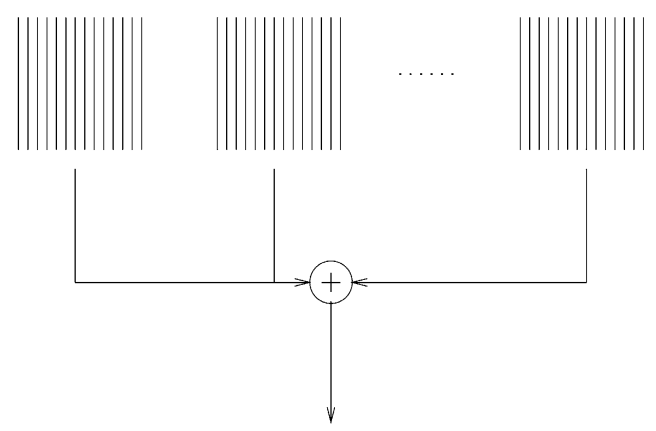
High resolution
image

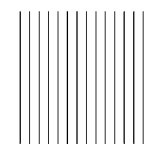

(b)

Fig. 1. The conventional STA approach (a) with sequential data acquisition in which only one transmitter is used in every transmission. The full aperture is synthesized in the beamforming. To the right, the FD. (b) Approach in which the full aperture is used in every transmission, and every transmitter only uses a narrow band of the available bandwidth. The signals are separated by a filterbank consisting of the matched filters corresponding to the excitation waveforms. The full spectrum of each transmitter is synthesized by transmitting several times and using different center frequencies every time.

mation can be decoded, making it subceptible to motion of the tissue.

In this paper, a novel method for exciting the entire aperture is proposed. The different transmitters are coded using narrow-band signals, which are disjointed in the frequency domain. Therefore, it is possible to extract the received information from each transmitter by matched filtration, and each waveform thereby represents a physical location on the aperture. The principle of the frequency division (FD) approach compared to conventional STA can be seen in Fig. 1. Note that it is still required to transmit more than once if the full bandwidth of the transducer is to be covered because the signals assigned to the different transmitters are narrow band. However, the full bandwidth of the transducer can be synthesized by assigning different signals to the transmitters from transmissionto-transmission and summing the filtered responses. The approach increases the SNR because more energy is transmitted in every transmission, and makes new information from all locations on the aperture available in every transmission.

In Section II the theory of the method will be described and analyzed. In Section III the beamforming involved in making the images will be explained briefly. Simulations will be shown in Section IV, in which the FD imaging technique will be compared to a conventional STA system with a sinusoid excitation. In Section V some measurements using the experimental RASMUS scanner [20] will be presented, and in Section VI concluding remarks will be given.

\section{THEORY}

The principles and the underlying theory concerning the frequency division imaging approach will be presented. In Section II-A the system model and the assumptions on this model will be described, in Section II-B the theoretical 
design of the excitation waveforms is discussed, continued by a practical explanation in Section II-C. The impact on SNR will be analyzed in Section II-D.

Consider an ultrasound system consisting of $K$ transmitters and $Q$ receivers. Let each transmitter be assigned an individual waveform $x_{p(k)}(t)$, where $p(k)$ represents the index of the waveform on the $k$ :th transmitter. If it is assumed that the system dynamics is linear, the received signal at the $q$ :th receiver $y_{q}(t)$ can be written $a^{2}$ :

$$
y_{q}(t)=\sum_{k=1}^{K} h_{k q}(t) \star x_{p(k)}(t),
$$

where $h_{k q}(t)$ represents the impulse response between the $k$ :th transmitter and the $q$ :th receiver. This impulse response is a function of the location of the transmitter $\vec{r}_{k}$, the location of the receiver $\vec{r}_{q}$ and the location of the scatters $\vec{r}_{m}$. For $M$ scatters, this impulse response would be:

$$
h_{k q}(t)=\sum_{m=1}^{M} h\left(\vec{r}_{k}, \vec{r}_{q}, \vec{r}_{m}, t\right),
$$

assuming that the medium is linear and consists of point scatters. The measured signal on the $q$ :th receiver, however, also is influenced by the system noise:

$$
r_{q}(t)=y_{q}(t)+v(t)
$$

where $v(t)$ is assumed to be a white, zero mean and Gaussian distributed process. The network structure of this system can be viewed in Fig. 2. The Fourier transform of (1) is given by:

$$
Y_{q}(f)=\sum_{k=1}^{K} H_{k q}(f) X_{p(k)}(f),
$$

where $H_{k q}(f)$ and $X_{p(k)}(f)$ are the Fourier transforms of $h_{k q}(t)$ and $x_{p(k)}(t)$, respectively.

The information about the system carried by each individual waveform has to be extracted from (1). One way of achieving this is to design the waveforms such that they are approximately disjointed in the frequency domain. This condition can be written as:

$$
X_{p}(f) X_{s}^{*}(f)=\left\{\begin{array}{ll}
\left|X_{p}(f)\right|^{2} & p=s \\
0 & p \neq s
\end{array} .\right.
$$

By dividing the available frequency band into a set of narrow bands and assigning one band to one specific transmitter, it, thus, is possible to use several transmitters at the same time and still be able to separate them at the receiver. Such a set of signals for a system with four transmitters can be seen in Fig. 3. Each transmitter is now

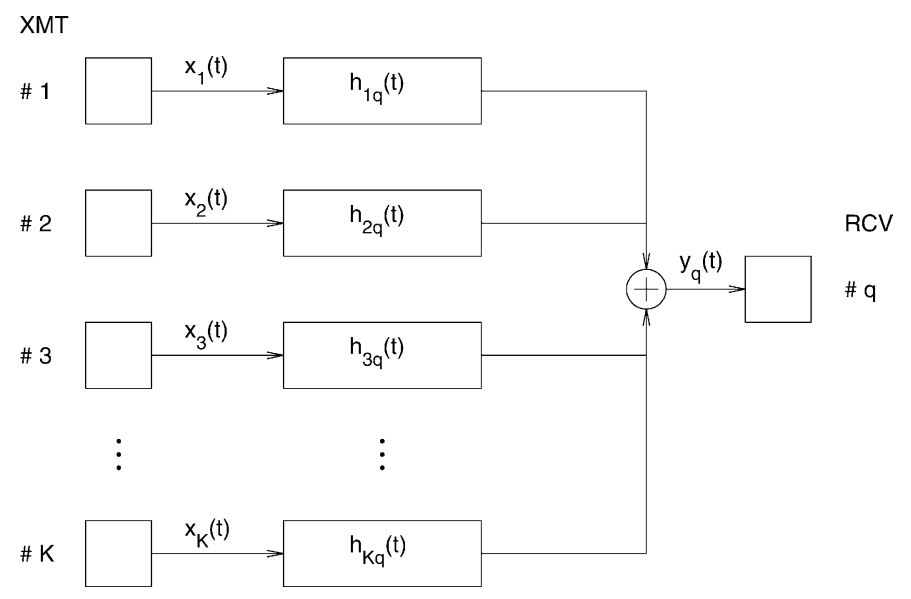

Fig. 2. The network structure of the input/output model of (1).

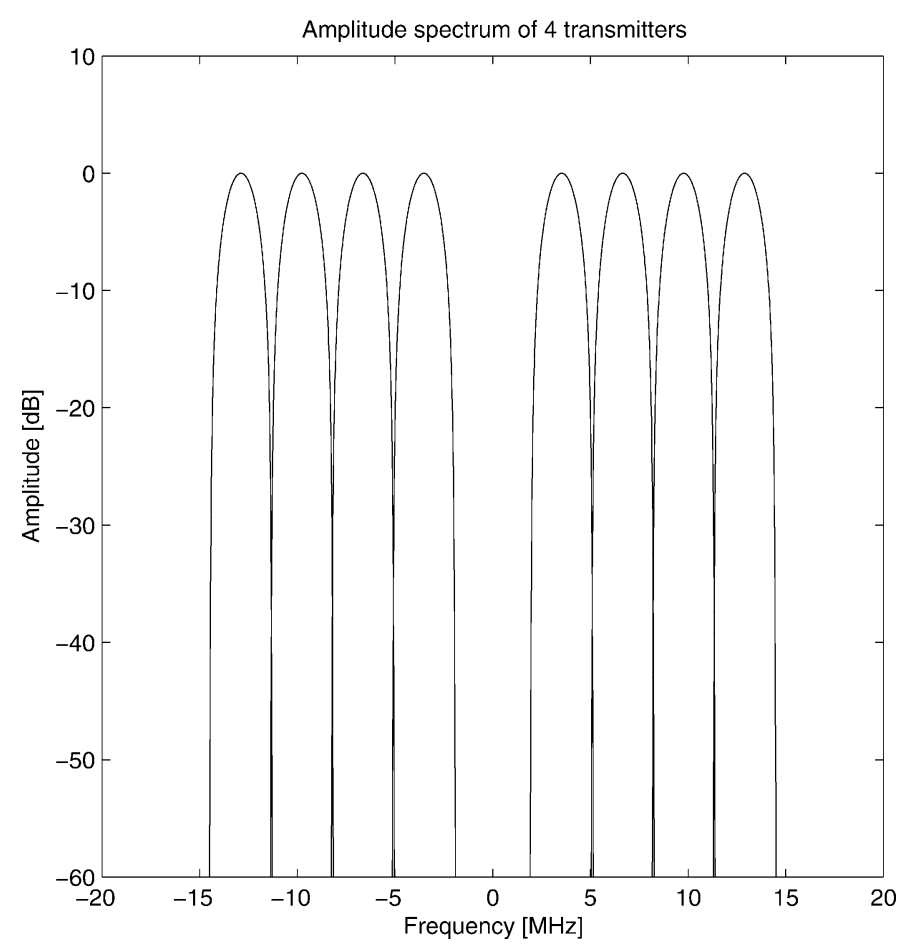

Fig. 3. A set of signals with the property specified in (5). The signals are weakly correlated and will remain so, even if subjected to different transfer functions representing tissue.

assigned an individual waveform. The received signal will be on the form as in (3), and the signal part of the received signal will be given by (1). If the attention is focused on the $l$ :th transmitter, the only term of interest is basically the contribution from this transmitter. The contributions from the other transmitters are considered to be interference. Because the signals have virtually no overlap in the frequency domain, it is possible to filter out all the interference by simply applying a filter matched to the waveform representing the transmitter of interest. Because the noise is assumed to be white, the matched filter for the

\footnotetext{
${ }^{2} \star$ denotes the convolution operator.
} 
$l:$ th transmitter is given by $[18] x_{p(l)}(-t)$, which has a frequency response $X_{p(l)}^{*}(f)^{3}$. The filtered version of (4) is then:

$$
\begin{aligned}
Y_{q}(f) X_{p(l)}^{*}(f) & =\left(\sum_{k=1}^{K} H_{k q}(f) X_{p(k)}(f)\right) X_{p(l)}^{*}(f) \\
& =H_{l q}(f)\left|X_{p(l)}(f)\right|^{2} .
\end{aligned}
$$

This result is independent of all other transmitters and can be treated as if transmitter $l$ had been transmitting alone. The result in (6), however, does not excite the full bandwidth of the transfer function $H_{l q}(f)$ and, thus, will not provide the best achievable resolution. By acquiring data over a number of transmissions, and changing the transmitted waveforms for the transmitters to cover the full frequency band of the transducer, this problem can be solved. The total contribution from the $l$ :th transmitter to the $q$ :th receiver over $N$ transmissions is now:

$$
\begin{aligned}
Y_{l q}(f) & =\sum_{n=1}^{N} H_{l q}(f)\left|X_{p(l, n)}(f)\right|^{2} \\
& =H_{l q}(f) \sum_{n=1}^{N}\left|X_{p(l, n)}(f)\right|^{2} .
\end{aligned}
$$

The subindex denoted by $p(l, n)$ is the number of the waveform transmitted on the $l$ :th transmitter in the $n:$ th transmitting event. By considering the different spectra in Fig. 3, it clearly can be seen that the summed frequency response does not produce a smooth function. This is unacceptable if artifacts are to be avoided. If a second set of signals is designed that is a frequency-shifted version of the first signal set, this problem can be circumvented. The requirement is that the summed, matched-filtered response results in a smooth spectrum that covers the full bandwidth of the transducer. The basic principle can be seen in Fig. 4. If it is assumed that the transfer function between the $l$ :th transmitter and the $q$ :th receiver $H_{l q}(f)$ is band-limited, and that the summed spectrum of the $N$ matched filtered waveforms is a constant function over this frequency band, then:

$$
Y_{l q}(f)=H_{l q}(f) .
$$

To cover the entire frequency band of the transducer for all transmitters, the number of waveforms $P=2 K$, which means that $2 K$ transmissions have to be made. It is imperative that signals from the first set are not transmitted at the same time as signals from the second set. This would destroy the orthogonality and it would not be possible to cancel the interference by matched filtering. Evidently some sort of pulsing strategy has to be devised. Let the set of $P$ waveforms be denoted:

$$
\left\{x_{p}(t)\right\}_{p=1}^{P},
$$

where the waveforms from $p=1$ to $p=K$ denotes the signals corresponding to the first signal set that are mutually

\footnotetext{
${ }^{3} X^{*}$ denotes complex conjugate of $X$.
}
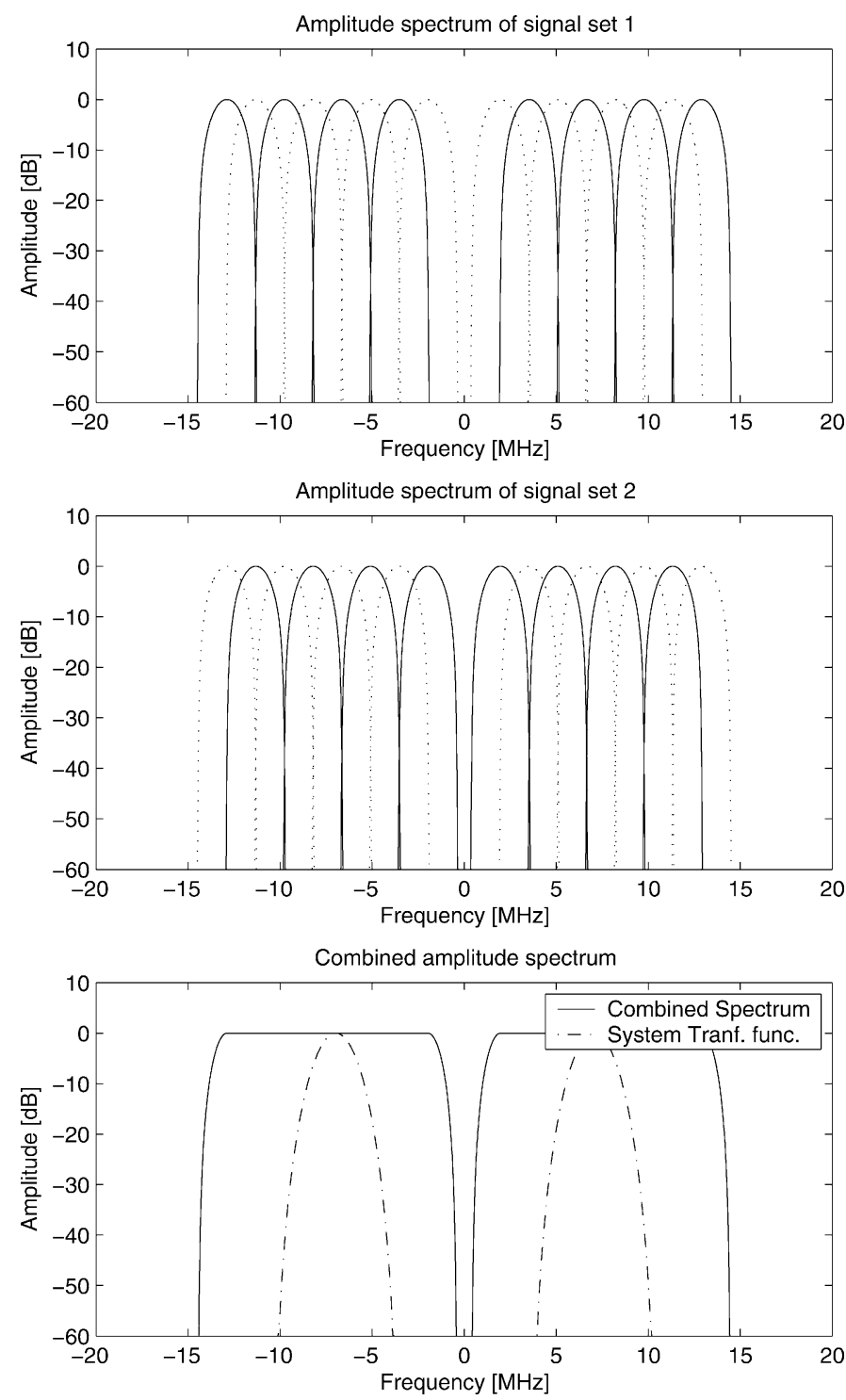

Fig. 4. The top figure shows the amplitude spectrum of the first signal set. The middle figure shows the amplitude spectrum of the second signal set. The bottom figure shows the matched filtered and summed amplitude response for these two signal sets.

orthogonal. The signals $p=K+1$ to $p=2 K$ corresponds to the signals from the second set, which are also mutually orthogonal, but not orthogonal to the signals in the first set. For a system with four transmitters and thus, eight different excitation waveforms a possible pulsing scheme is given in Table I. The filtered and summed time (axial) response of the signals corresponding to the spectra in Fig. 4 can be examined in Fig. 5 .

Because an ultrasound system has a maximum excitation voltage, the limiting factor concerning the transmitted energy is the amplitude of the waveforms. Therefore, it is important to design the waveforms such that the full excitation voltage can be applied over the full duration of the signal. Thus, the transmitted signals should 
TABLE I

Firing Sequence for the Total Signal Set.

\begin{tabular}{ccccccccc}
\hline$p(k, n)$ & \multicolumn{10}{c}{$n$} \\
$k$ & 1 & 2 & 3 & 4 & 5 & 6 & 7 & 8 \\
\hline 1 & 1 & 2 & 3 & 4 & 5 & 6 & 7 & 8 \\
2 & 4 & 1 & 2 & 3 & 8 & 5 & 6 & 7 \\
3 & 3 & 4 & 1 & 2 & 7 & 8 & 5 & 6 \\
4 & 2 & 3 & 4 & 1 & 6 & 7 & 8 & 5 \\
\hline
\end{tabular}

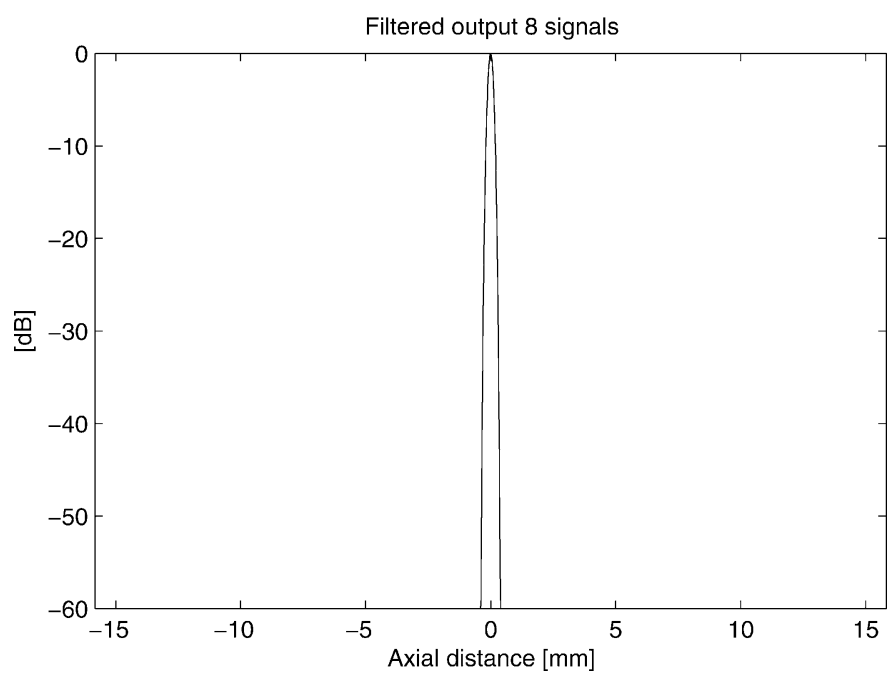

Fig. 5. The time response of the filtered and summed signals corresponding to Fig. 4 convolved with the impulse response of a $7 \mathrm{MHz}$ transducer. Mutually orthogonal signals were summed to simulate simultaneous transmission.

have long duration with fairly constant amplitude to maximize SNR, narrow bandwidth, and low sidelobes in the frequency domain to minimize inter-band interference. An obvious choice would be to use amplitude weighted narrowband linear frequency modulated signals (chirps) with a very slow FM slope. A chirp with bandwidth $B$, center frequency $f_{p}$, duration $T$, and temporal amplitude modulation function $a(t)$ is defined as:

$x_{p}(t)=a(t) \sin \left\{2 \pi\left[\left(f_{p}-\frac{B}{2}\right) t+\frac{B}{2 T} t^{2}\right]\right\}, 0 \leq t \underset{(10)}{t}$.

The corresponding matched filter is simply the time reciprocal of the excitation waveform. For the examples in this paper, the duration $T$ of the waveforms is chosen to be $20 \mu \mathrm{s}$. The bandwidth of each individual waveform was $1 \mathrm{MHz}$. The signals were weighted using a Tukey window with $75 \%$ tapering to reduce ripple in the combined spectrum such that temporal sidelobes were minimized [8]. Sixteen waveforms were produced, organized in two subsets of eight signals. The distance in the frequency domain between two adjacent signals in the same subset was chosen to be $1 \mathrm{MHz}$. This value was chosen in order to produce a smooth, combined spectrum and get an acceptable interband interference level. The first signal in the first subset had a center frequency of $3.3 \mathrm{MHz}$, and the first signal
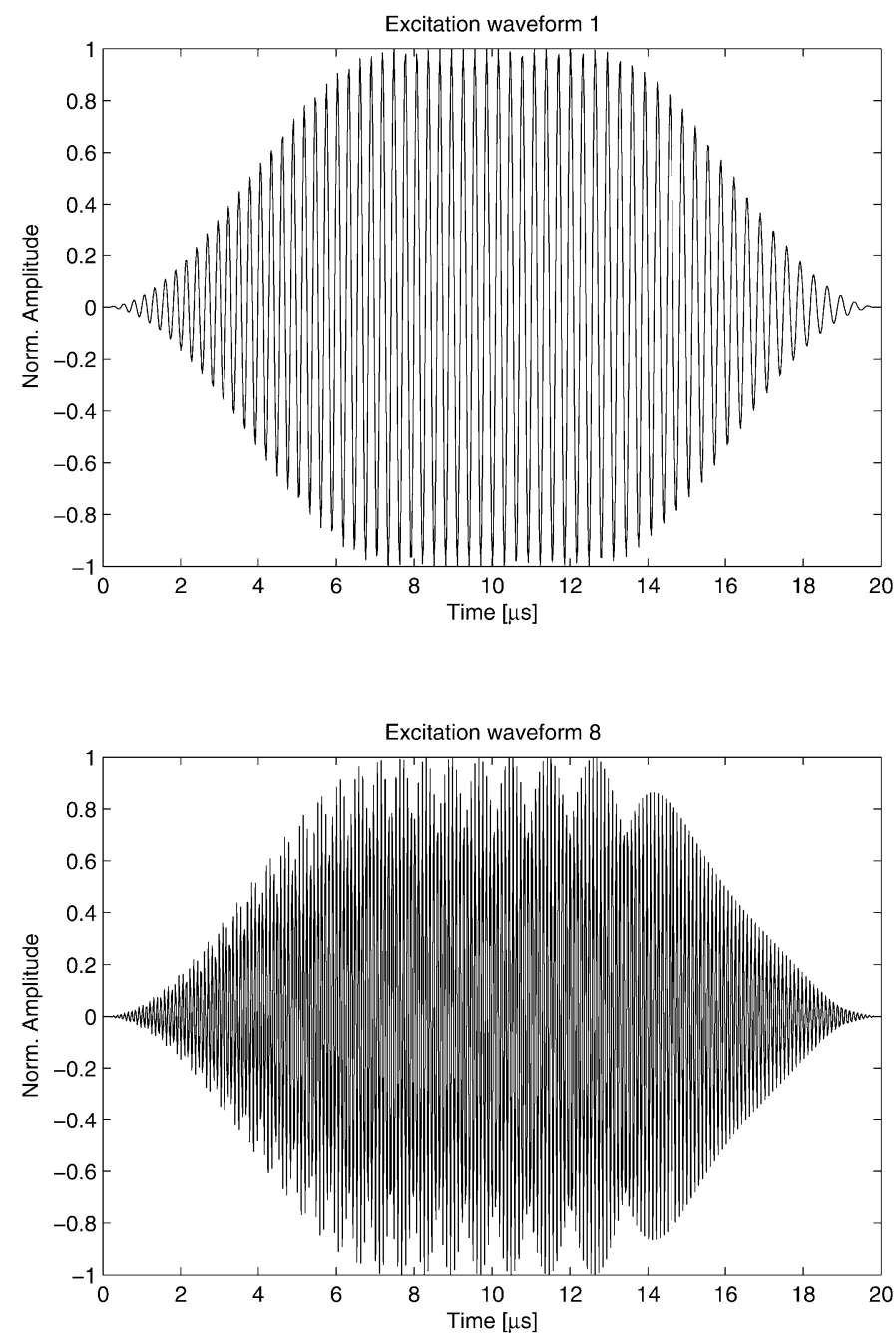

Fig. 6. Top, the first (smallest center frequency, 3.3 MHz). Bottom, last (largest center frequency, $10.3 \mathrm{MHz}$ ) signals from the first signal subset.

in the second subset had a center frequency of $3.8 \mathrm{MHz}$. Two signals from the first subset can be seen in Fig. 6, in which the top most figure is the signal corresponding to the smallest center frequency, and the bottom figure is the signal with the largest center frequency. The cross talk between two signals (the $p$ :th and the $s$ :th signal) will be defined as:

$$
\gamma_{p, s}=\frac{\int\left|x_{p}(t) \star x_{s}(t)\right|^{2} d t}{\int\left|x_{p}(t) \star x_{p}(t)\right|^{2} d t}
$$

The cross talk between two neighboring bands in the same signal subset, $\gamma_{p, p+1}$ was $-46 \mathrm{~dB}$. The inter-band interference produced a ripple in the combined spectrum with an amplitude of $0.1 \mathrm{~dB}$. The resulting signal amplitude spectra can be seen in Fig. 7. The time response for the combined spectrum was computed and can be seen in Fig. 8 . The temporal sidelobes at $\pm 12 \mathrm{~mm}$ is a result of the ripple in the combined spectrum. The influence of these sidelobes will be analyzed using simulations in Section IV. 

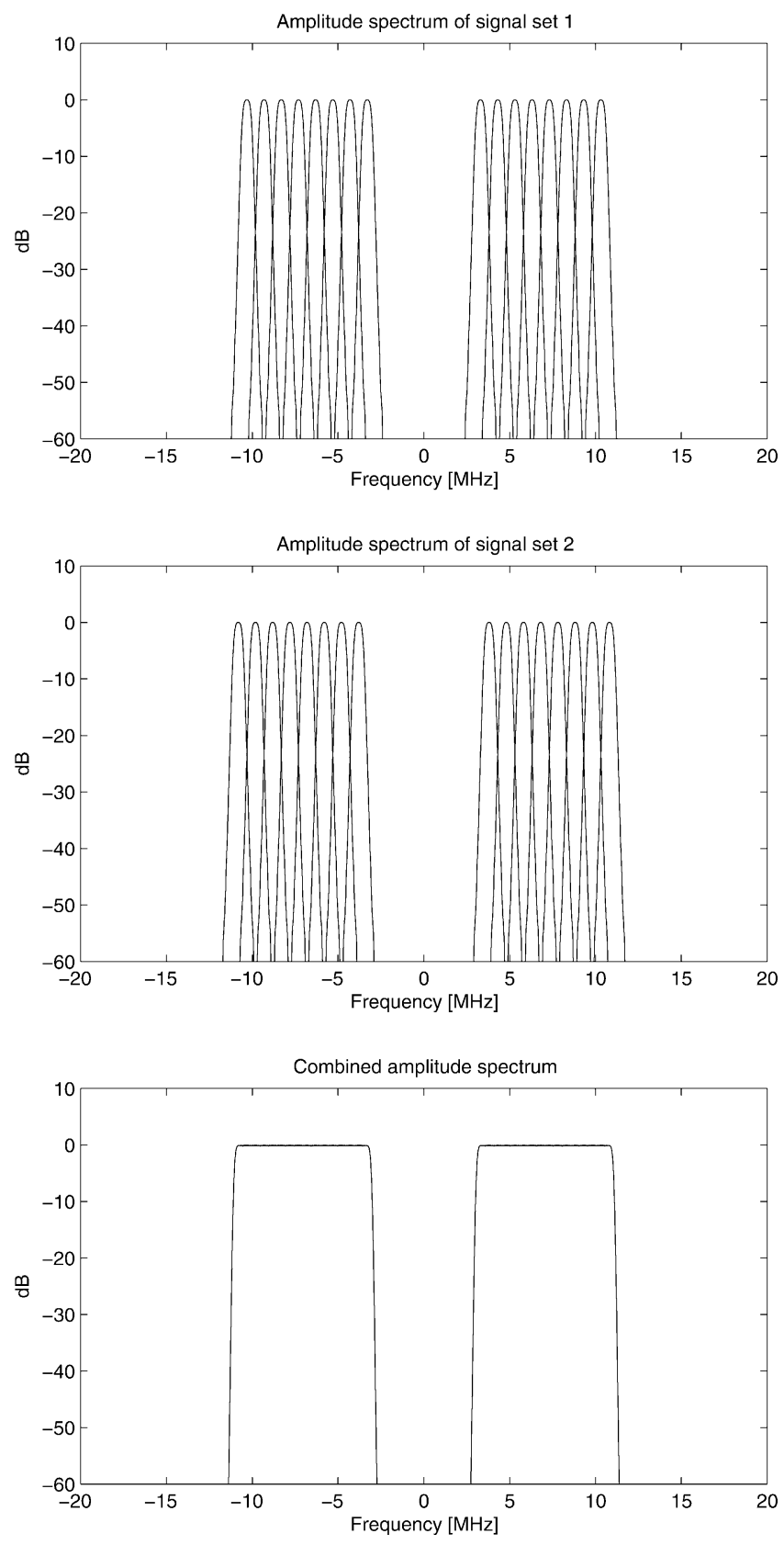

Fig. 7. The resulting amplitude spectra for the 16 narrow-band FM signals. The (top) waveform has a center frequency of $3.3 \mathrm{MHz}$. The (center) bandwidth of the individual waveforms is $1 \mathrm{MHz}$. The (bottom) is a combined spectrum.

In this section, the influence on the peak SNR will be discussed. Consider a setup in which the investigated object is a single point scatterer in an nonattenuating medium. The received signal will then simply be a timeshifted and scaled version of the transmitted waveform plus the system noise, which is assumed to be white, zero mean, and Gaussian distributed. The noise also is assumed to be uncorrelated from transmission-totransmission and from transducer element-to-transducer element. Consider first the conventional synthetic trans-

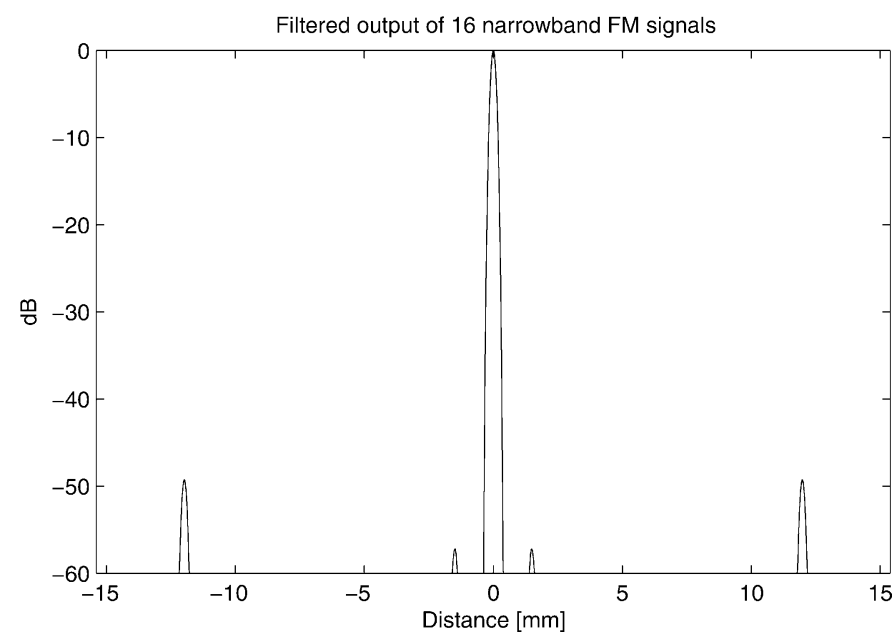

Fig. 8. The time response of the combined spectrum for the 16 signals. Signals from the two subsets were added to simulate simultaneous transmission.

mission aperture method with sequential data acquisition. The transmitted waveform is denoted $x(t)$ and its Fourier transform is $X(f)$ with energy $E_{\mathrm{REF}}$. The received signal from one transmission then will be (omitting time shift and scaling for simplicity):

$$
r(t)=x(t)+v(t)
$$

where $v(t)$ is the system noise with power $P_{v}$. The matched filtered output of (12) will be:

$$
r(t) \star x(-t)=x(t) \star x(-t)+v(t) \star x(-t) .
$$

The peak SNR is given by:

$$
\mathrm{SNR}=\frac{\left.|x(t) \star x(-t)|_{t=t_{\max }}\right|^{2}}{E\left[|v(t) \star x(-t)|^{2}\right]},
$$

where $E[(\cdot)]$ is the expectancy operator applied to $(\cdot)$. The expression inside the absolute value of the numerator in (14) is given by [19]:

$$
\left.x(t) \star x(-t)\right|_{t=t_{\max }}=\int_{-\infty}^{\infty}|X(f)|^{2} d f=E_{\mathrm{REF}},
$$

whereas the denominator will be:

$$
E\left[|v(t) \star x(-t)|^{2}\right]=\int_{-\infty}^{\infty} P_{v}|X(f)|^{2} d f=P_{v} E_{\mathrm{REF}} .
$$

Assuming $K$ transmitters, $2 K$ transmissions are made for the sake of fareness compared to the FD approach. It it is assumed that the summing of the data originating from the different transmissions is coherent. The final SNR is then:

$$
\mathrm{SNR}_{\mathrm{REF}}=\frac{(2 K)^{2} E_{\mathrm{REF}}^{2}}{2 K P_{v} E_{\mathrm{REF}}}=\frac{2 K E_{\mathrm{REF}}}{P_{v}} .
$$


The attention is now turned to the FD imaging method. The narrow band waveforms $\left\{x_{p}(t)\right\}_{p=1}^{P}$ all have the energy $E_{\mathrm{FD}}$. The response for one transmitter is constructed using $P=2 K$ transmissions. The received signal for one transmitter and one transmission is:

$$
r_{p}(t)=x_{p}(t)+v_{p}(t) .
$$

The matched filtered response is:

$$
r_{p}(t) \star x_{p}(-t)=x_{p}(t) \star x_{p}(-t)+v_{p}(t) \star x_{p}(-t),
$$

giving the total response of:

$$
r_{m f}(t)=\sum_{p=1}^{2 K} x_{p}(t) \star x_{p}(-t)+v_{p}(t) \star x_{p}(-t),
$$

which in turn yields the filtered signal peak:

$$
\begin{aligned}
\left.\sum_{p=1}^{2 K} x_{p}(t) \star x_{p}(-t)\right|_{t=t_{\max }} & = \\
& \sum_{p=1}^{2 K} \int_{-\infty}^{\infty}\left|X_{p}(f)\right|^{2} d f=2 K E_{\mathrm{FD}},
\end{aligned}
$$

and the filtered noise power:

$$
\begin{aligned}
& E\left[\left|\sum_{p=1}^{2 K} v_{p}(t) \star x_{p}(-t)\right|^{2}\right]= \\
& \sum_{p=1}^{2 K} \int_{-\infty}^{\infty} P_{v}\left|X_{p}(f)\right|^{2} d f=2 K P_{v} E_{\mathrm{FD}}
\end{aligned}
$$

Once again, coherent summing of the data from the different transmitters is assumed. The noise from transmission-to-transmission also is assumed to be uncorrelated, which gives the total SNR:

$$
\mathrm{SNR}_{\mathrm{FD}}=\frac{(2 K K)^{2} E_{\mathrm{FD}}^{2}}{2 K K P_{v} E_{\mathrm{FD}}}=\frac{2 K^{2} E_{\mathrm{FD}}}{P_{v}} .
$$

The ratio of SNR's, thus, will be:

$$
\frac{\mathrm{SNR}_{\mathrm{FD}}}{\mathrm{SNR}_{\mathrm{REF}}}=K \frac{E_{\mathrm{FD}}}{E_{\mathrm{REF}}} .
$$

\section{BEAMFORMING}

This section describes the beamforming, which has been applied to properly focus the data. When the data from one specific transmitter is available, either by applying the FD method and filtering out the data from a specific transmitter or using only one transmitter at a time, it is possible to exactly determine where on the aperture the acoustic wave was transmitted, and where it was received. This allows the synthetic aperture focusing technique described in $[2]$ to be applied.

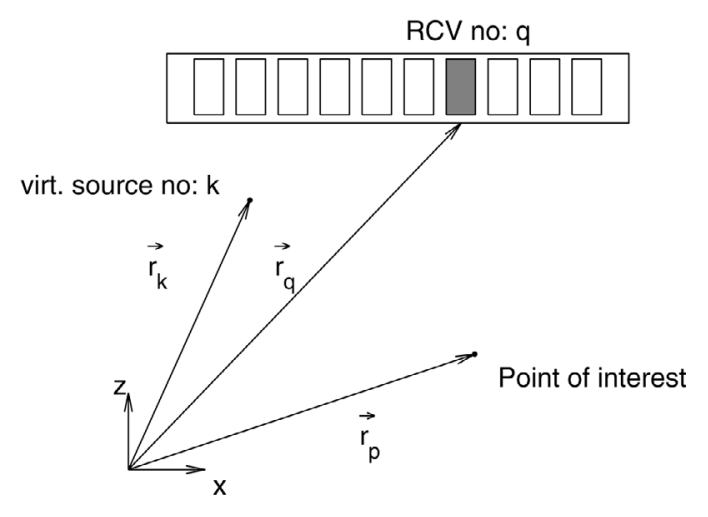

Fig. 9. Principles of synthetic aperture focusing for a point $p$ for the $k$ :th transmitter and the $q$ :th receiver.

Because the system has access to defocused transmissions from all transmitters on the aperture, it is possible to focus in any arbitrarily chosen point of interest. The amplitude in a point $p$ in the image is given by:

$$
H\left(\vec{r}_{p}\right)=\sum_{k=1}^{K} \sum_{q=1}^{Q} g_{q} h_{k q}\left(t_{p k q}\right)
$$

where $g_{q}$ is a weighting function (apodization) over the receiving aperture, and $h_{k q}(t)$ is the received (and possibly filtered) echo on the $q$ :th receiver originating from the $k$ :th transmitter. Here $t_{p k q}$ is the time corresponding to the geometrical travel distance from the $k$ :th transmitter to the point $p$ and back to the $q$ :th receiver:

$$
t_{p k q}=\frac{\left\|\vec{r}_{p}-\vec{r}_{k}\right\|+\left\|\vec{r}_{q}-\vec{r}_{p}\right\|}{c}
$$

where $c$ is the speed of sound, and $\vec{r}_{p}-\vec{r}_{k}$ is the vector from the transmitter to the point $p$, and $\vec{r}_{q}-\vec{r}_{p}$ is the vector from the point $p$ to the receiver as indicated in Fig. 9. Summing contributions from all transmissions for all receiving elements then gives both dynamic transmit and receive focusing.

\section{Simulations}

The method was tested using the virtual ultrasound scanner software Field II [20], [21]. A 7 MHz, 128 element transducer was simulated. The kerf was $0.035 \mathrm{~mm}$, pitch $0.208 \mathrm{~mm}$, and height $4.5 \mathrm{~mm}$. The transmitting aperture was created by grouping and focusing 64 of the central transducer elements so that eight virtual sources were formed. The bandwidth of the transducer limited the maximum number of active transmitters to eight, which in turn resulted in that only the 64 central elements were used, so the spacing between the virtual sources was kept on a reasonable level. Each virtual source consisted of eight transducer elements that were focused $2.5 \mathrm{~mm}$ in front of the transducer surface. The receiving aperture used all 128 elements with a Hanning weighting. The sampling frequency was $120 \mathrm{MHz}$. The transducer impulse response was set 
TABLE II

Resolution as a Function of Depth, FD Imaging.

\begin{tabular}{ccccc}
\hline & \multicolumn{2}{c}{ Axial res. mm } & \multicolumn{2}{c}{ Lateral res. mm } \\
\cline { 2 - 5 } Depth $(\mathrm{mm})$ & $-3 \mathrm{~dB}$ & $-6 \mathrm{~dB}$ & $-3 \mathrm{~dB}$ & $-6 \mathrm{~dB}$ \\
\hline 30 & 0.192 & 0.278 & 0.245 & 0.403 \\
35 & 0.189 & 0.272 & 0.270 & 0.429 \\
40 & 0.185 & 0.268 & 0.291 & 0.464 \\
45 & 0.184 & 0.265 & 0.334 & 0.522 \\
50 & 0.184 & 0.265 & 0.400 & 0.601 \\
55 & 0.184 & 0.264 & 0.449 & 0.653 \\
60 & 0.183 & 0.265 & 0.484 & 0.728 \\
\hline
\end{tabular}

TABLE III

Resolution as a Function of Depth, Conventional STA IMAGING.

\begin{tabular}{ccccc}
\hline & \multicolumn{2}{c}{ Axial res. $\mathrm{mm}$} & \multicolumn{2}{c}{ Lateral res. $\mathrm{mm}$} \\
\cline { 2 - 5 } Depth $(\mathrm{mm})$ & $-3 \mathrm{~dB}$ & $-6 \mathrm{~dB}$ & $-3 \mathrm{~dB}$ & $-6 \mathrm{~dB}$ \\
\hline 30 & 0.201 & 0.292 & 0.268 & 0.433 \\
35 & 0.196 & 0.286 & 0.274 & 0.446 \\
40 & 0.195 & 0.283 & 0.324 & 0.508 \\
45 & 0.194 & 0.282 & 0.377 & 0.575 \\
50 & 0.194 & 0.281 & 0.435 & 0.637 \\
55 & 0.194 & 0.281 & 0.473 & 0.712 \\
60 & 0.195 & 0.283 & 0.530 & 0.788 \\
\hline
\end{tabular}

to be a single cycle sinusoid at $7 \mathrm{MHz}$ with a temporal Hanning weighting.

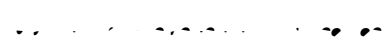

All transmitters were used in every transmission. Two sets of signals were created. Each set consisted of eight narrow band FM signals. The first band had a center frequency of $3.3 \mathrm{MHz}$, and the spacing in the frequency domain was $1 \mathrm{MHz}$. The signals were $20 \mu \mathrm{s}$ long. The target was seven point scatterers starting at $30 \mathrm{~mm}$ from the transducer surface with a $5 \mathrm{~mm}$ spacing. The result can be seen in Fig. 10. The resolution as a function of depth can be seen in Table II. Note that the axial sidelobes, which occurred in the time response in Section II-C, are not present in the image in Fig. 10.

As a reference, a conventional synthetic aperture transmission scheme was used. One virtual source was used in every transmission. The excitation was a single-cycle sinusoid with a center frequency of $7 \mathrm{MHz}$ with a temporal Hanning weighting. The target was once again seven point scatterers starting at $30 \mathrm{~mm}$ from the transducer surface with a $5 \mathrm{~mm}$ spacing. The result can be seen in Fig. 10. The resolution as a function of depth can be seen in Table III. From Tables II and III it can be concluded that the FD approach yields the same resolution as a normal STA system with a sinusoid excitation. The lateral sidelobe level was also the same, below $-45 \mathrm{~dB}$.

\section{EXPERIMENTS}

The FD imaging method has been tested in an experimental setup, and the results were compared to other methods. The ultrasound research scanner RASMUS [22] was used for the measurements, with a BK8804 $7 \mathrm{MHz}$ linear array transducer (B-K Medical A/S, Herlev, Denmark). The FD method was compared to a system that used a single-cycle sinusoid excitation and to a system with a linear frequency-modulated signal as excitation.

The transmitting and receiving aperture was the same for all three methods; 64 active elements were used as the transmitting aperture. The reason for using only the central 64 tranducer elements was the same as in Section IV. The transmitting elements were grouped and focused such that eight virtual sources were formed. The focusing point was $2.5 \mathrm{~mm}$ in front of the transducer surface. Each virtual source was created by eight transducer elements. The RASMUS system only has access to 64 elements as receiving aperture, but 128 receiving elements were emulated by multiplexing. The receiving aperture was weighted by a Hanning window. All three methods used the synthetic aperture focusing technique described in [2]. The main difference is that the FD method used all active elements in transmit, and the two other methods used only one virtual source at a time.

The excitation in this case was a single-cycle sinusoid with a center frequency of $7 \mathrm{MHz}$ corresponding to the center frequency of the transducer, with a temporal Hanning weighting.

$$
\cdots \quad \ldots, \ldots,(\ldots,)
$$

The linear frequency modulated excitation waveform had a center frequency of $7 \mathrm{MHz}$, duration $20 \mu \mathrm{s}$, and a bandwidth of $75 \%$ of the center frequency. The waveform was tapered using a Tukey window with $20 \%$ tapering. The compression filter was the corresponding matched filter version of the excitation waveform mismatched using a Chebyshev window with relative sidelobe attenuation of $70 \mathrm{~dB}$. The temporal window applied to derive the mismatched filter is used in order to suppress axial sidelobes [8]. The excitation waveform and the corresponding mismatched filter can be seen in Fig. 11.

All virtual sources were excited in every transmission. This was done by assigning the waveforms derived in Section II-C to the different transmitters.

Three measurements were done using a string phantom from Dansk Fantomservice (Jylling, Denmark) to evaluate 

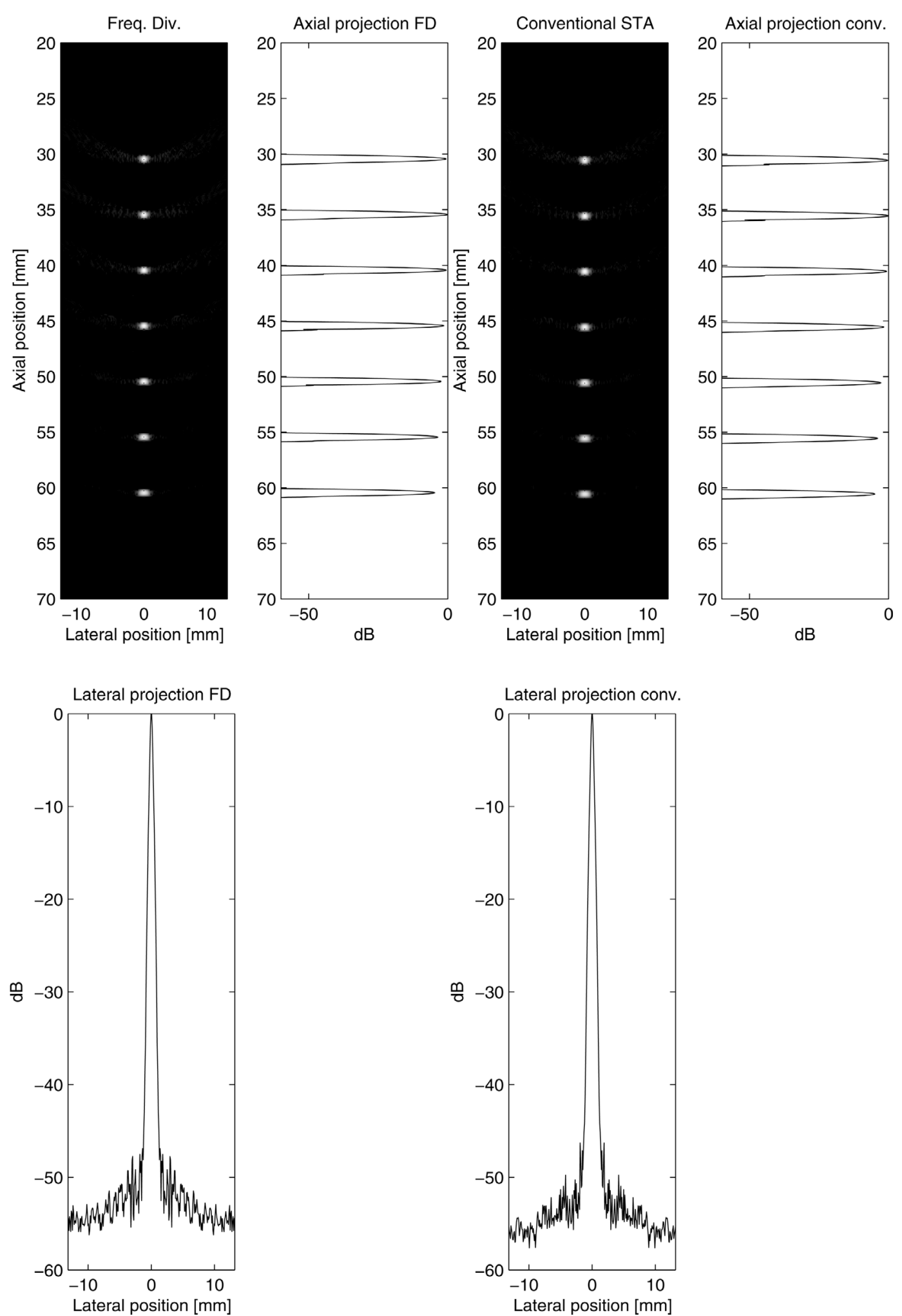

Fig. 10. The simulation of the response for the seven point scatterers. The figures to the left show the response for the frequency division method with eight virtual sources and 16 bands. The figures to the right show the response for a conventional synthetic aperture transmission approach using a single-cycle sinusoid at $7 \mathrm{MHz}$ as excitation. 

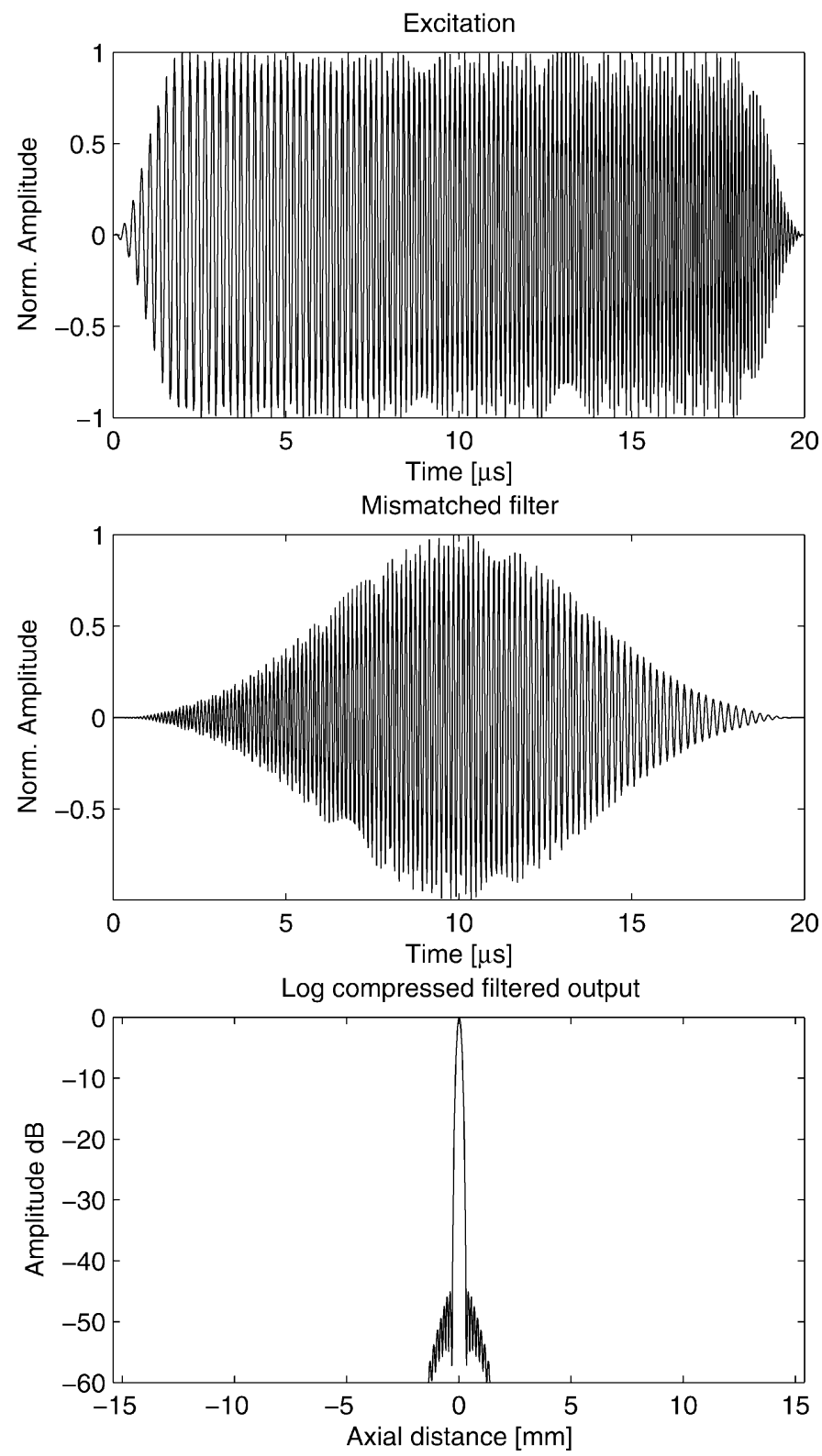

Fig. 11. The top figure is the excitation waveform. The center figure is the corresponding mismatched filter. The bottom figure is envelope of the filtered output. Note that the rangelobes will be further suppressed by the transducer impulse response.

resolution. The result from the three measurements can be seen in Fig. 12. The FD approach improves the penetration depth by $3.5-4 \mathrm{~cm}$ compared to the system using the single-cycle sinusoid excitation, and the penetration depth is improved by $1-2 \mathrm{~cm}$ compared to the system using the chirp excitation. The axial and lateral resolution as a function of depth can be seen in Tables IV and V for the FD approach and chirp excitation, respectively. The axial resolution is somewhat better for the setup using the chirp for depth 22-42 mm, and the FD approach has better axial resolution for depths $52-62 \mathrm{~mm}$. The lateral resolution in all cases is in favor of the setup using the chirp excitation, but the difference is so small it is concluded that the lateral resolution is comparable for the two different excitation schemes.
TABLE IV

Resolution as a Function of Depth, FD Imaging.

\begin{tabular}{ccc}
\hline & Axial res. mm & Lateral res. mm \\
\cline { 2 - 3 } Depth $(\mathrm{mm})$ & $-6 \mathrm{~dB}$ & $-6 \mathrm{~dB}$ \\
\hline 22 & 0.568 & 0.568 \\
32 & 0.590 & 0.567 \\
42 & 0.557 & 0.684 \\
52 & 0.520 & 0.851 \\
62 & 0.534 & 0.989 \\
\hline
\end{tabular}

TABLE V

Resolution as a Function of Depth, Chirp Sta Imaging.

\begin{tabular}{ccc}
\hline & Axial res. mm & Lateral res. mm \\
\cline { 2 - 3 } Depth $(\mathrm{mm})$ & $-6 \mathrm{~dB}$ & $-6 \mathrm{~dB}$ \\
\hline 22 & 0.437 & 0.519 \\
32 & 0.450 & 0.561 \\
42 & 0.460 & 0.679 \\
52 & 0.633 & 0.830 \\
62 & 0.666 & 0.923 \\
\hline
\end{tabular}

The SNR also was measured and compared to the results derived in Section II-D. The same setup was used as in the phantom measurements. However, the setup using a single-sinusoid excitation was ignored. Instead the chirp excitation was used as a reference. A speckle phantom from Dansk Fantom service M571 was used (90.6\% water, $9.4 \%$ glycerol (85\%), $0.2 \% \mathrm{Na}$-benzonat, $0.1 \% \mathrm{~K}_{2}-$ EDTA). Only speckle was measured. One hundred images were acquired using the setup with a chirp as excitation waveform. These images were added pair-wise to emulate the same number of transmissions as in the FD approach. Next, 50 images were acquired using the FD approach. The two equally large sets of images were averaged in order to get an estimate of the signal component in the images:

$$
S(i, j)=\frac{1}{N} \sum_{n=1}^{N} H_{n}(i, j),
$$

where $H_{n}(i, j)$ is a sample in a specific high-resolution image originating from one data acquisition (full image constructed from several firings). The resulting signal component was subtracted from the images to get an estimate of the noise component in the images:

$$
N(i, j)=H_{n}(i, j)-S(i, j)
$$

The SNR for a specific depth then was computed for the center line in an image as:

$$
\operatorname{SNR}(d)=\frac{\sum_{i=i_{d}-L+1}^{i_{d}} S\left(i, j_{c}\right)^{2}}{\sum_{i=i_{d}-L+1}^{i_{d}} N\left(i, j_{c}\right)^{2}},
$$



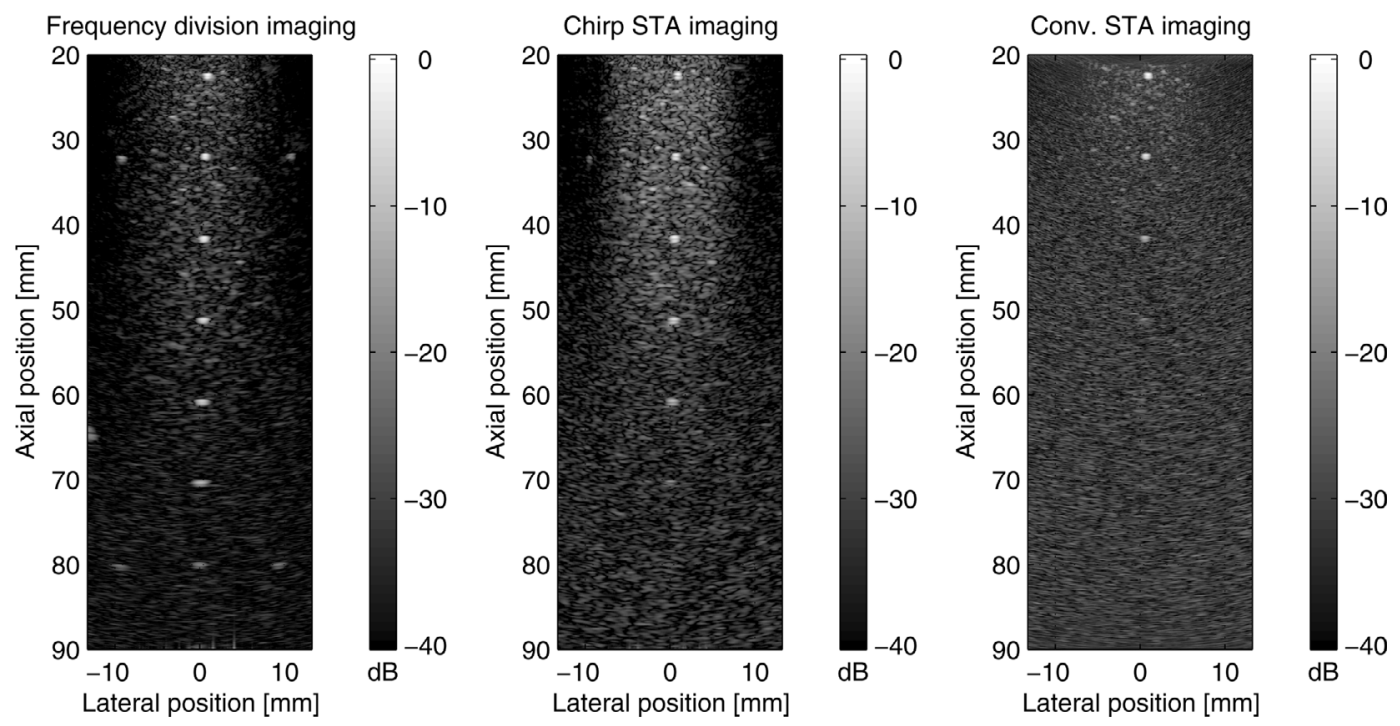

Fig. 12. The left image is the image produced by the FD approach using 16 frequency bands. The middle image is the image produced by the chirp specified in Section V-B. The right image is the image resulting from a single-sinusoid excitation with a center frequency of 7 MHz.

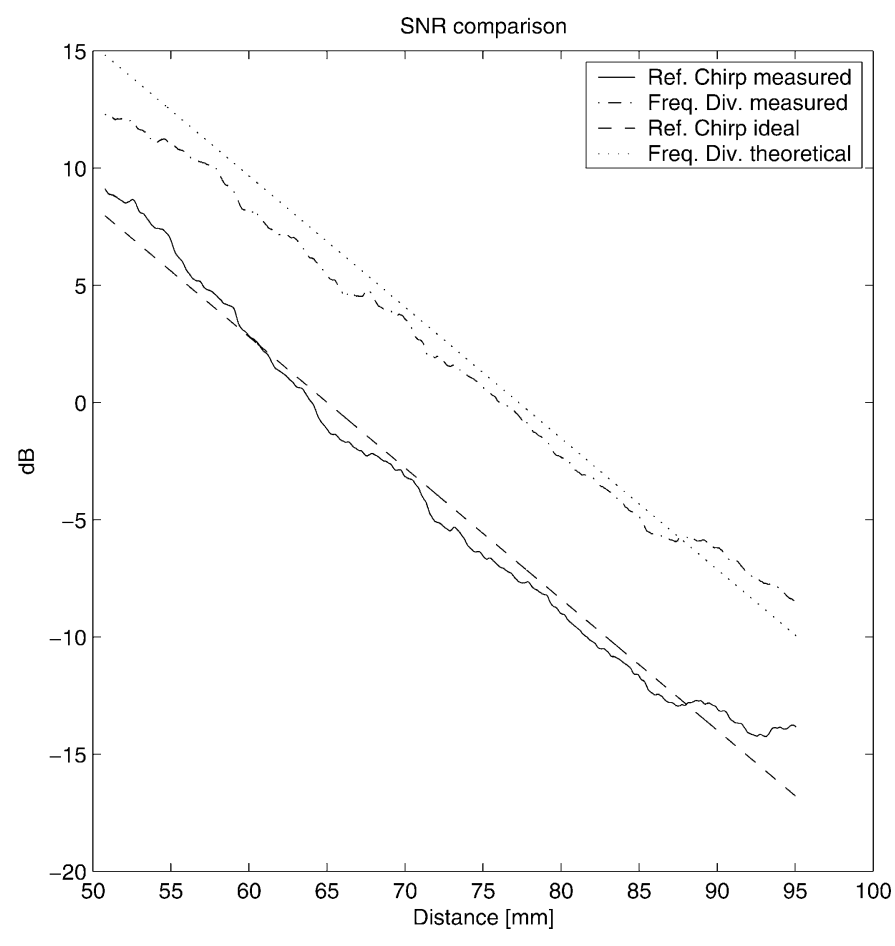

Fig. 13. The SNR as a function of depth. The solid line is the measured SNR for the reference experiment using a chirp excitation. The dashed line is the same data but after linear regression has been applied. The dashed-dotted line is the SNR measurement using the FD approach. The dotted line is the theoretical SNR for the FD approach using the chirp excitation as a reference.

where $i_{d}$ corresponds to the row in the image that represents the depth $d, i_{c}$ is the center line and $L$ is the segment length over which the SNR estimate is computed. The segment length was chosen to be 200 samples, which corresponds to $3.85 \mathrm{~mm}$ in the phantom, assuming that the speed of sound is $1540 \mathrm{~m} / \mathrm{s}$.

The result can be seen in Fig. 13. The data originating from the measurement using the chirp was fitted to a straight line using linear regression. Because the number of transmitters is $K=8$ and the ratio of signal energies $\left(E_{\mathrm{FD}} / E_{\text {chirp }}\right)=0.61$, according to $(24)$, the gain in SNR should be $6.86 \mathrm{~dB}$. The theoretical gain in SNR can be seen as the dotted line in Fig. 13. The theoretically computed gain in SNR agrees with the measured gain in SNR.

\section{Conclusions}

A method for exciting several transmitters simultaneously and separating them in receive has been proposed. Compared to the traditional way of acquiring data in synthetic transmit aperture imaging, this method has the advantage that it can use all transmitters in every transmission. This means that the transmitted energy will be increased, and that the SNR will be increased. The major advantage of the approach is that the acquired information from the multiple transmitters can be separated from each other instantaneously, once the data has been received. It is not necessary as in Hadamard or Golay coding to use multiple transmissions before it is possible to decode the data. The proposed approach, thus, is not influenced by motion effects in the decoding step. However, the broadband synthesis will be influenced by motion. It is unclear how this will affect the method. Subsequently, a thorough analysis of motion artifacts is needed to fully understand the implications of motion. Furthermore, a temporal coding concept also has been deployed in which the different narrow-band signals are linearly frequency modulated, such that the instantaneous frequency of the signals increases linearly with time. This enables the system to use the full excitation voltage for a longer time. These two factors combined means that the number of transmissions can be decreased and the same SNR can be achieved, which is an advantage in cases of rapid tissue movement or in flow imaging. The method has been evaluated using the simulation tool Field II, in which it was compared to traditional 
synthetic aperture acquisition using a single-cycle sinusoid excitation. The resolution and contrast were found to be comparable in the two different situations.

A measurement in an attenuating string phantom also has been performed. The frequency division approach was compared to a conventional STA acquisition approach using both a chirp and a sinusoidal excitation. The penetration depth was found to be increased by $1-2 \mathrm{~cm}$ compared to the chirp excitation and $3.5-4 \mathrm{~cm}$ compared to sinusoidal excitation. The setup using the sinusoidal excitation was excluded in the resolution comparison because of the poor penetration depth. Concerning axial and lateral resolution, the FD approach and the conventional STA using a chirp was found to yield approximately the same performance. A SNR measurement was also performed. The FD approach was compared to the STA setup using a chirp excitation. The improvement in SNR was found to be approximately $6.5 \mathrm{~dB}$, which agrees with the theoretically derived results.

\section{ACKNOWLEDGMENT}

The authors are grateful for the comments from the reviewers.

\section{REFERENCES}

[1] S. Bennett, D. K. Peterson, D. Corl, and G. S. Kino, "A realtime synthetic aperture digital acoustic imaging system," in Proc. 10th Int. Symp. Acoust. Imag., vol. 10, P. Alais and A. F. Metherell, Eds. 1982, pp. 669-692.

[2] S. I. Nikolov, "Synthetic aperture tissue and flow ultrasound imaging," Ph.D. dissertation, Ørsted•DTU, Technical University of Denmark, Lyngby, Denmark, 2001.

[3] J. W. Goodman, Introduction to Fourier Optics. 2nd ed. New York: McGraw-Hill, 1996.

[4] M. O'Donnell, "Coded excitation system for improving the penetration of real-time phased-array imaging systems," IEEE Trans. Ultrason., Ferroelect., Freq. Contr., vol. 39, pp. 341-351, 1992.

[5] T. X. Misaridis, K. Gammelmark, C. H. Jørgensen, N. Lindberg, A. H. Thomsen, M. H. Pedersen, and J. A. Jensen, "Potential of coded excitation in medical ultrasound imaging," Ultrasonics, vol. 38, pp. 183-189, 2000.

[6] T. X. Misaridis, M. H. Pedersen, and J. A. Jensen, "Clinical use and evaluation of coded excitation in B-mode images," in Proc. IEEE Ultrason. Symp., 2000, pp. 1689-1693.

[7] T. X. Misaridis and J. A. Jensen, "An effective coded excitation scheme based on a predistorted FM signal and an optimized digital filter," in Proc. IEEE Ultrason. Symp., 1999, pp. 15891593.

[8] T. Misaridis, "Ultrasound imaging using coded signals," Ph.D. dissertation, Ørsted•DTU, Technical University of Denmark, Lyngby, Denmark, 2001.

[9] M. O'Donnell and L. J. Thomas, "Efficient synthetic aperture imaging from a circular aperture with possible application to catheter-based imaging," IEEE Trans. Ultrason., Ferroelect., Freq. Contr., vol. 39, pp. 366-380, 1992.

[10] M. Karaman, P. C. Li, and M. O'Donnell, "Synthetic aperture imaging for small scale systems," IEEE Trans. Ultrason., Ferroelect., Freq. Contr., vol. 42, pp. 429-4422, 1995.

[11] C. Passmann and H. Ermert, "A 100-MHz ultrasound imaging system for dermatologic and ophthalmologic diagnostics," IEEE Trans. Ultrason., Ferroelect., Freq. Contr., vol. 43, pp. 545-552, 1996.

[12] C. H. Frazier and W. D. O'Brien, "Synthetic aperture techniques with a virtual source element," IEEE Trans. Ultrason., Ferroelect., Freq. Contr., vol. 45, pp. 196-207, 1998.
[13] K. L. Gammelmark and J. A. Jensen, "Multi-element synthetic transmit aperture imaging using temporal encoding," in Proc. SPIE: Prog. Biomed. Opt. Imag., 2002, vol. 3, pp. 25-36.

[14] K. L. Gammelmark and J. A. Jensen, "Multielement synthetic transmit aperture imaging using temporal encoding," IEEE Trans. Med. Imag., vol. 22, no. 4, pp. 552-563, 2003.

[15] M. H. Pedersen, K. L. Gammelmark, and J. A. Jensen, "Preliminary in-vivo evaluation of convex array synthetic aperture imaging," in Proc. SPIE-Progress in Biomedical Optics and Imaging, 2004, pp. 33-43.

[16] R. Y. Chiao, L. J. Thomas, and S. D. Silverstein, "Sparse array imaging with spatially encoded transmits," in Proc. IEEE Ultrason. Symp., 1997, pp. 1679-1682.

[17] T. Misaridis and J. A. Jensen, "Space-time encoding for high frame rate ultrasound imaging," Ultrasonics, vol. 40, pp. 593597, 2002.

[18] R. Y. Chiao and L. J. Thomas, "Synthetic transmit aperture using orthogonal golay coded excitation," in Proc. IEEE Ultrason. Symp., 2000, pp. 1469-1472.

[19] S. Haykin, Communication Systems. New York: Wiley, 2001.

[20] J. A. Jensen and N. B. Svendsen, "Calculation of pressure fields from arbitrarily shaped, apodized, and excited ultrasound transducers," IEEE Trans. Ultrason., Ferroelect., Freq. Contr., vol. 39, pp. 262-267, 1992.

[21] J. A. Jensen, "Field: A program for simulating ultrasound systems," in Med. Biol. Eng. Comp., 10th Nordic-Baltic Conf. Biomed. Imag., vol. 4, suppl. 1, pt. 1, pp. 351-353, 1996.

[22] J. A. Jensen, O. Holm, L. J. Jensen, H. Bendsen, S. I. Nikolov, B. G. Tomov, P. Munk, M. Hansen, K. Salomonsen, J. Hansen, K. Gormsen, H. M. Pedersen, and K. L. Gammelmark, "Ultrasound research scanner for real-time synthetic aperture image acquisition," IEEE Trans. Ultrason., Ferroelect., Freq. Contr., vol. 52, no. 5, May 2005, pp. 881-891.

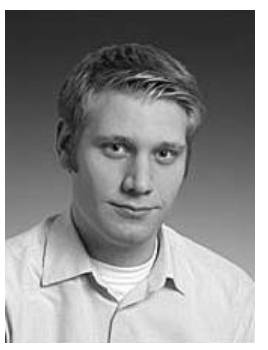

Fredrik Gran earned his M.Sc. degree in engineering physics from Lund University, Sweden in 2002 and the Ph.D. degree in electrical engineering from the Technical University of Denmark (DTU) in 2005. He is currently working as a post-doctoral researcher at the Center for Fast Ultrasound imaging at DTU.

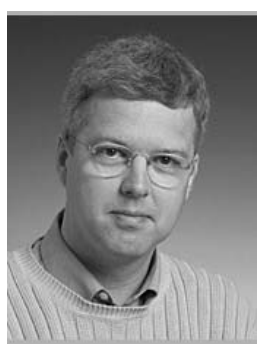

Jørgen Arendt Jensen (M'93-SM'02) earned his Master of Science in electrical engineering in 1985 and the Ph.D. degree in 1989, both from the Technical University of Denmark. He received the Dr.Techn. degree from the university in 1996. He has published a number of papers on signal processing and medical ultrasound and the book Estimation of Blood Velocities Using Ultrasound, Cambridge University Press, in 1996.

He is also developer of the Field II simulation program. He has been a visiting scientist at Duke University, Stanford University, and the University of Illinois at Urbana-Champaign. He is currently full professor of Biomedical Signal Processing at the Technical University of Denmark at Ørsted•DTU and head of Center for Fast Ultrasound Imaging. He has given courses on blood velocity estimation at both Duke University and University of Illinois and teaches biomedical signal processing and medical imaging at the Technical University of Denmark. He has given several short courses on simulation, synthetic aperture imaging, and flow estimation at international scientific conferences. $\mathrm{He}$ is also the co-organizer of a new biomedical engineering education program offered by the Technical University of Denmark and the University of Copenhagen. His research is centered around simulation of ultrasound imaging, synthetic aperture imaging and blood flow estimation, and constructing systems for such imaging. 